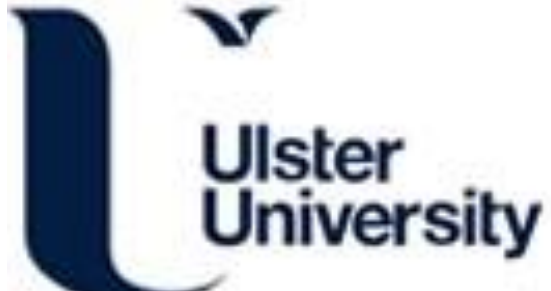

\section{Denoising RF defibrillator waveforms for intracardiac atrial substrate impedance characterisation using digital filtering techniques}

Escalona, OJ., Walsh, PR., Rababah, AS., Kodoth, V., \& Manoharan, G. (2015). Denoising RF defibrillator waveforms for intracardiac atrial substrate impedance characterisation using digital filtering techniques. In Unknown Host Publication (Vol. 51, pp. 1329-1332). Springer. https://doi.org/10.1007/978-3-319-19387-8_324

Link to publication record in Ulster University Research Portal

\section{Published in:}

Unknown Host Publication

Publication Status:

Published (in print/issue): 30/10/2015

DOI:

10.1007/978-3-319-19387-8_324

\section{Document Version}

Publisher's PDF, also known as Version of record

\section{General rights}

Copyright for the publications made accessible via Ulster University's Research Portal is retained by the author(s) and / or other copyright owners and it is a condition of accessing these publications that users recognise and abide by the legal requirements associated with these rights.

\section{Take down policy}

The Research Portal is Ulster University's institutional repository that provides access to Ulster's research outputs. Every effort has been made to ensure that content in the Research Portal does not infringe any person's rights, or applicable UK laws. If you discover content in the Research Portal that you believe breaches copyright or violates any law, please contact pure-support@ulster.ac.uk. 


\title{
Denoising RF defibrillator waveforms for intracardiac atrial substrate impedance characterisation using digital filtering techniques
}

\author{
O.J. Escalona ${ }^{1}$, P.R. Walsh ${ }^{1}$, A.S.Rababah ${ }^{1}$,V. Kodoth ${ }^{2}$ and G. Manoharan ${ }^{2}$ \\ ${ }^{1}$ Centre for Advanced Cardiovascular Research, University of Ulster, Newtownabbey, Northern Ireland \\ ${ }^{2}$ The Heart Centre, Royal Victoria Hospital, Belfast, Northern Ireland
}

\begin{abstract}
Intra-cardiac impedance (ICI) is one of the major determinants of success during internal cardioversion of atrial fibrillation(AF) and is consequently of significant importance in the development of more effective cardioversion therapies. In this study, the voltage and current waveform delivered during internal cardioversion of patients with persistent AF treated using a novel low-tilt rectilinear waveform (generated by a radiofrequency (RF) defibrillator) and following a step up energy protocol $(50 \mathrm{~V}-300 \mathrm{~V}$ in $50 \mathrm{~V}$ steps) were recorded. However, within the clinical environment these waveforms are typically compromised with electrical noise. The objective of this work was to investigate if it was possible to develop an algorithm, using digital signal processing (DSP)in the MATLAB environment, to denoise the AF defibrillation voltage and current waveforms by applying spectral analysis and digital filtering techniques.

Preliminary results indicated that spectral analysis of the processed rectilinear waveform signals allowed estimation of the most appropriate cutoff frequency as $1125 \mathrm{~Hz}-2250 \mathrm{~Hz}$. Initial results also indicated that application of a Hanning windowing was more appropriate than Blackman windowing in the spectral preservation of the rectilinear waveform signals. Furthermore, for this particular type of waveform, noise reduction using a 7th order Butterworth filter design was found to be more efficacious than a 200 order FIR digital filter.

In conclusion, within this explorative study, the processing algorithm developed was effective for denoising voltage and current signals recorded during cardioversion and therefore provides a useful tool for characterisation of intra-cardiac impedance during cardioversion.
\end{abstract}

Keywords - Inracardiac impedance, atrial fibrillation, RF defibrillator, low-tilt rectilinear waveforms, internal cardioversion.

\section{INTRODUCTION}

Atrial fibrillation (AF) is the most common cardiac arrhythmias and accounts for 30\%-40\% of all cardiac arrhythmia related hospital admissions [1]. It is one of the leading causes of stroke, affects an estimated 33.5 million people worldwideand is responsible for increased morbidity and mortality. Atrial fibrillation can be treated via pharmacological cardioversion, surgical ablation or electrical cardioversion; depending on several clinical factors such as patient history, AF type and risk of thrombus. Over the past decade, a strong focus has developed on intra-cardiac electrical cardioversion; the introduction of electrodes transvenously to deliver an electrical shock directly to the heart. However, although clinically safe and effective, outcome is dependent on several factors such as waveform shape, position of the electrodes and intra-cardiac impedance (ICI). In 2006, Walsh, S. et al. showed that an asymmetric $6 / 6 \mathrm{~ms}$ low tilt biphasic waveforms is the optimum waveform for transvenous cardioversion of atrial fibrillation [2]. Another study revealed that the coronary sinus right atrium position is preferred over pulmonary artery right atrium position since it lower the energy requirement threshold required for successful shock [3]. In addition, a pilot study of a low-tilt biphasic waveform for transvenous cardioversion of atrial fibrillation concluded that the low-tilt biphasic waveform was more successful for the internal cardioversion of both persistent and induced AF in patients [4]. However, there is a paucity of studies that have examined the dynamic variation of intra-cardiac impedance within the time domain as a determinant factor to outcome during internal cardioversion of atrial fibrillation $[5,6]$. In order to analyse and investigate ICI dynamics during internal cardioversion of AF, voltage and current waveforms are recorded between the two defibrillation electrodes. Nevertheless, within the clinical environment these signals typically suffer significant noise; from sources such as the RF power source, ECG, EMG, EEG, etc. The objective of this work was to investigate if it was possible to develop an algorithm, using digital signal processing (DSP) in the MATLAB environment, to denoise the AF defibrillation voltage and current waveforms by applying spectral analysis and digital filtering techniquesand thereby facilitate the further extraction of potentially valuable intra-cardiac dynamic impedance (ICI) metrics.

\section{Methods}

Eight patients with persistence AF who would benefit from internal electrical cardioversion were recruited for this study at the Royal Victoria Hospital Belfast. Patients were 
treated clinically by using anticoagulant and adequate sedation prior to atrial cardioversion. A St. Jude 6 French Inquiry $^{\mathrm{TM}}$ internal cardioversion catheter was positioned under fluoroscopic guidance in the right atrium and the distal coronary sinus [5]. Monophasic very-low-tilt rectilinear waveform (M-VLTR) was selected and a step up voltage protocol from $50 \mathrm{~V}$ to $300 \mathrm{~V}$ in $50 \mathrm{~V}$ steps was used for therapy. Simultaneously, voltage and current waveforms were digitally recorded at $250 \mathrm{kHz}$ sampling frequency using a digital oscilloscope (Tektronix TDS 3014B) and a current probe (Fluke 80i-110s) [7]. This data was then exported from .csv to MSExcel format and loaded into a MATLAB database for further manipulation.

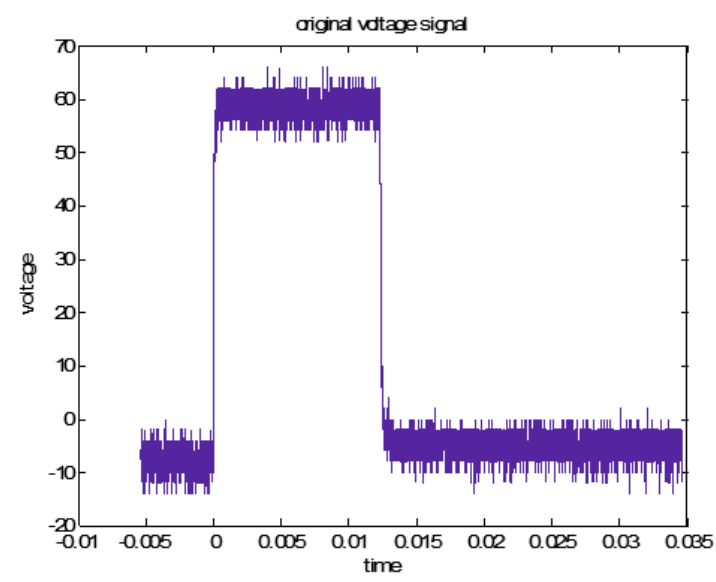

Fig. 1 Noisy voltage rectilinear waveform recorded by the digital oscilloscope (RF defibrillator setting of 50V)

As is self-evident in figure 1, voltage and current signals are noisy; with some type of signal processing necessary to distinguish signal from noise. Two techniques were used to find the appropriate cutoff frequency for separation of signal from background noise. First, an ideal monophasic very low tilt rectilinear shock signal was produced using MATLAB functions. Then, post application of a Hanning window, the frequency spectrum of this ideal signal was generated. Next, the clinical (noisy) waveforms captured (voltage and current) were windowed using both Hanning and Blackman techniques; with the associated frequency spectrums generated. After defining the appropriate cut-off frequency, two MATLAB built in filter design commands, butter and FIR1, were applied to assess impact on noise reduction. Finally, the time domain intra-cardiac impedance signal was calculated from filtered voltage and current waveforms via application of Equation 1.

$$
\operatorname{Resistance}(\Omega)=\frac{\operatorname{Voltage}(\mathrm{V})}{\operatorname{Current}(\mathrm{A})}
$$

From a top level perspective, the MATLAB algorithm was developed to automatically handle the large data files recorded during defibrillation therapy. Patient number and case number were used to automate loading of the associated defibrillation voltage and current waveforms and deduce the sampling frequency; as previously described based on a-priori rectilinear pulse width $(12 \mathrm{~ms})$. The algorithm is designed to then select the desired waveform segment (part of the rectilinear signal in figure 1 at time between $2 \mathrm{~ms}$ and $10 \mathrm{~ms}$ ) and automatically denoise, generate and plots the filtered waveforms. Finally, the algorithm calculates the dynamic resistance (ICI) and associated parameters.

\section{ResUlts}

With reference to figure 1 , the algorithm was designed to extract a signal segment of $8 \mathrm{~ms}$ duration (from $2 \mathrm{~ms}$ to $10 \mathrm{~ms}$ ) from each of the entire voltage waveform captured; with $2 \mathrm{~ms}$ from the beginning and $2 \mathrm{~ms}$ from the end of the signal discarded to ensure that only the stable part of the signal (with no fast transitions) is sampled. Removal of this unstable part of the signal significantly reduces the amount of unwanted frequency components in the resulting spectrum due to the sudden change (from approximately zero to just below $60 \mathrm{~V}$ and vice versa) during the rising and falling edges within the shock waveform. Both an ideal shock signal (generated by defibrillator before interacting with patient heart tissue) and noisy (clinically acquired) signals were multiplied with Hanning and Blackman windows for spectral analysis. Because Hanning window has narrower main lobe (figure 2), it is more beneficial in this type of spectral analysis as the frequency spectrum of this signal contains very closely spaced features.
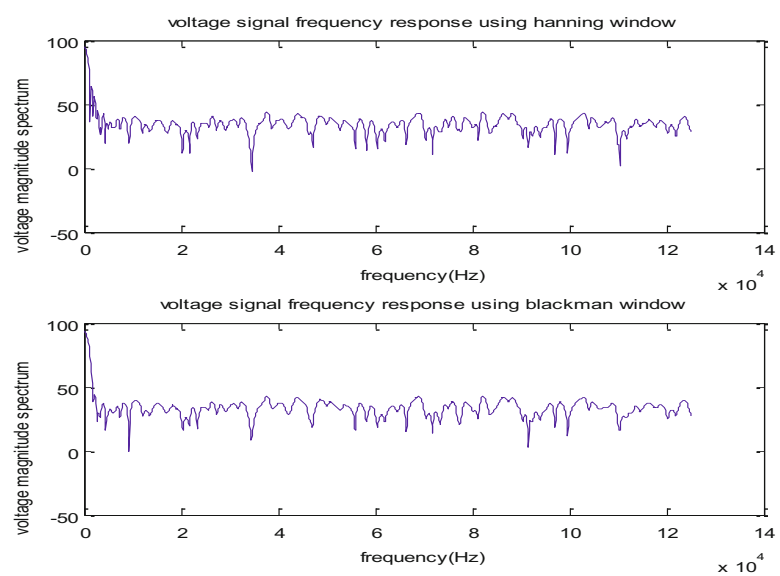

Fig. 2 Frequency spectrum of voltage signal after windowing with Hanning (top) and Blackman (bottom) windows. 
Based on this analysis and knowing that the frequency spectrum of ideal signal does not contain frequency components higher than $500 \mathrm{~Hz}(\mathrm{Fs}=250 \mathrm{kHz})$, it is clear that the cutoff frequency should be greater than $500 \mathrm{~Hz}$ in order to prevent distortion. However, tissue response to applied energy is known to add higher frequency components to the measured waveforms. As a result, denoising filters with different cutoff frequencies starting from a normalized frequency of 0.01 which corresponds to $1125 \mathrm{~Hz}$ were applied. Combining all these data together and investigating the effect of cutoff frequency on the desired signals, it is deduced that cutoff frequency between 0.01 and 0.02 would be the best choice. Subsequent, to the spectral analysis undertaken, two built in MATLAB filter functions were used to filter the signals, and the filtered signals were compared to select which is better for noise reduction of these signals.

FIR filter: After analyzing the frequency response of 200 order low pass FIR filter with a cutoff frequency of 0.015 , it is noticed that the pass band region was not flat as required. This leads to reduction in the amplitude of some components of the signal. In other words, this filter may distort the filtered signal significantly. In addition, the transition band of the filter is wide. As a result, some undesired high frequency signals may pass to become part of the filtered signal. Overcoming these problems necessitates increasing the order of the filter to not more than 200 was determined empirically. However, in doing so, undesired amplification of the signal in some regions and alteration of the characteristics of signal results. The following MATLAB code excerpt was used for the final FIR filter design implemented:

wn_voltage $=0.015 ; \%$ Normalized cutoff frequency.

$\%$ To calculate sampling frequency:

fs_voltage $=1 /($ time_all_voltage(2)-time_all_voltage(1));

fftpoints_number_voltage $=$ length(voltage_desired) $* 5$;

b2 $=$ fir $1(\overline{2} 00, w n$ voltage,'low'); \% 200 order FIR filter.

filtered_voltage_fir=filter(b2,1,voltage_desired);

$[\mathrm{H} 2, \mathrm{f2}]=$ freqz(b2,1,fftpoints_number_voltage,fs_voltage $)$;

$\operatorname{plot}(\mathrm{f} 2, \mathrm{abs}(\mathrm{H} 2))$;

title('frequency response of fir filter (cutoff freq is 0.015 )');

xlabel('frequency');ylabel('amplitude gain');

Butterworth filter: A 7th order Butterworth low pass filter with a cutoff frequency of 0.015 offers advantage in that it is a maximally flat magnitude filter. In other words, this filter passes the required frequencies without changing the characteristic amplitudes. However, trying to increase the order of the filter results in an unstable response in the passband region. Hence, this filter has the best performance at 7 th order. Flatness of the passband and acceptable transition width made this filter perform better in filtering this specific type of cardioversion waveform using an RF defibrillator. Again, the following MATLAB code excerpt was used for the final Butterworth filter design implemented:

wn_voltage $=0.015 ; \%$ normalized cutoff frequency.

fs_voltage $=1 /($ time_all_voltage(2)-time_all_voltage(1));

$\%$ to calculate sampling frequency.

fftpoints number_voltage $=$ length(voltage_desired $) * 5$;

$\% 7^{\text {th }}$ order Butterworth filter:

$[\mathrm{b} 1, \mathrm{a} 1]=\operatorname{butter}(7, \mathrm{wn}$ voltage $)$;

filtered_voltage_butter=filter(b1,a1,voltage_desired);

$[\mathrm{H} 1, \mathrm{fl}]=$ freqz $\left(\mathrm{b} 1, \mathrm{a} 1, \mathrm{fftpoints} \_n u m b e r \_v o l t a g e, f \mathrm{f} \_v o l t a g e\right)$; $\operatorname{plot}(\mathrm{fl}, \mathrm{abs}(\mathrm{H} 1))$;

title('frequency response of Butt filtr (cutoff freq is 0.015)'); xlabel('frequency');ylabel('amplitude gain');
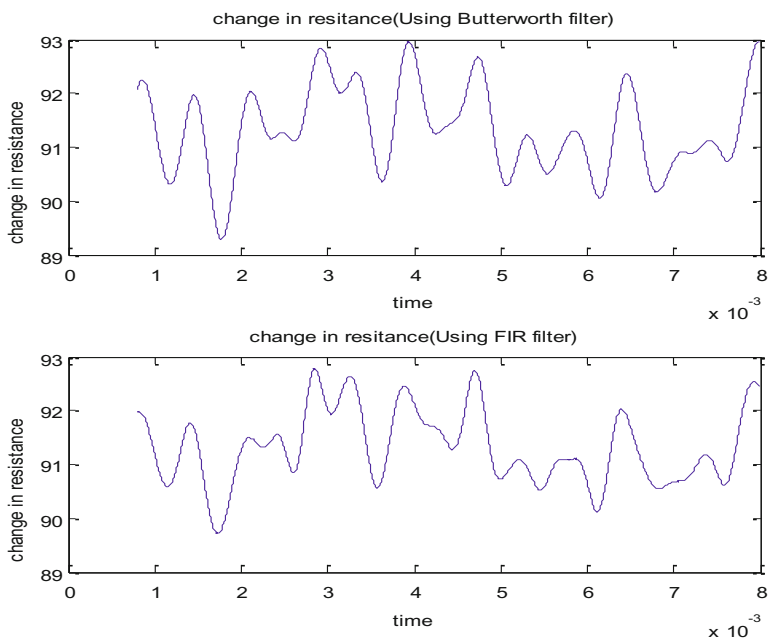

Fig. 3 Change in resistance at time segment between $1 \mathrm{~ms}$ and $8 \mathrm{~ms}$ after using Butterworth (top) and FIR filter (bottom).

Comparison between the outcome of chosen FIR and Butterworth filters: Both filters were effective in noise reduction as shown in figure 3. However, the FIR filter passes unwanted high frequency components as is evident at time $\mathrm{t}=$ $2-3 \mathrm{~ms}$ and $\mathrm{t}=6-7 \mathrm{~ms}$. Moreover, it reduces the peak to peak amplitude of the signal; because the response is not flat in the passband region. Again, this is self evident at time $\mathrm{t} \leq 2 \mathrm{~ms}$ and at $\mathrm{t}=6.5 \mathrm{~ms}$; for both diagrams in figure 3 .

\section{Discussion}

Noise reduction can be implemented using several techniques. Goodfellow, J. et al. assessed the efficacy of a novel wavelet based dominant atrial fibrillatory frequency (DAFF) 
estimation technique on twenty patients who underwent internal cardioversion at Royal Victoria Hospital, Belfast and showed that the wavelet techniques offered improved performance at attenuating noise than a conventional average template subtraction [8]. However, in this study, another technique based on digital filters is used to similarly reduce the noise in the ECG signals recorded during internal cardioversion. This signal contains significant noise generated from various electronic and physiologic sources; including RF power supply, ECG, EEG, EMG etc. Since this noise contains energy spread over a wide range of frequencies and levels it becomes a challenging task to distinguish between signal and noise and to define the most suitable cutoff frequency that will facilitate noise reduction without alteration to the key characteristics of the clinical signal. Consequently, accurate spectral analysis is required. In comparing the outcome of chosen FIR and Butterworth filter, both filters were effective in noise reduction as shown in figure 3 but the FIR filter passes unwanted high frequency components; as was evident in the time domain between $\mathrm{t}=2-3 \mathrm{~ms}$ and $\mathrm{t}=6-7 \mathrm{~ms}$. Moreover, as stated, it reduces the peak to peak amplitude of the signal because it is not flat in the passband region; again, this being evident at time $t \leq 2 \mathrm{~ms}$ and at $t=6.5 \mathrm{~ms}$ for both diagrams presented in figure 3 .

\section{v. Conclussions}

The objective of this work was to investigate the possibility to develop an algorithm, using digital signal processing (DSP) in the MATLAB environment, to denoise the AF defibrillation voltage and current waveforms by applying spectral analysis and digital filtering techniques. Specifically, the MATLAB algorithm was developed to automatically handle the large data files recorded during defibrillation therapy. Patient number and case number were used to automate loading of the associated defibrillation voltage and current waveforms and deduce the sampling frequency; based on a-priori rectilinear pulse width $(12 \mathrm{~ms})$. The algorithm was designed to then select the desired waveform segment (part of the rectilinear signal at time between $2 \mathrm{~ms}$ and $10 \mathrm{~ms}$ ) and denoises, generates and plots the filtered waveforms. Analyzing the spectrum of the processed rectilinear waveform signals provided the most appropriate normalized cutoff frequency estimation. The study results provided this being between 0.01 and 0.02 which corresponds to $1125 \mathrm{~Hz}-2250 \mathrm{~Hz}$. The evaluation results also indicated that Hanning windowing was more appropriate than Blackman windowing in the spectral analysis preservation of the rectilinear waveform signals. Furthermore, noise reduction of these signals using a 7th order Butterworth filter design was found to be more efficacious in denoising than a 200 order FIR digital filter. Overall, the processing algorithm developed and applied to RF defibrillator waveforms was effective for denoising voltage and current signals and provides a potentially useful tool for studying large number of patient cases in the characterisation of intra-cardiac impedance and its potential relation to other factors such as the outcome of cardioversion treatment and defibrillation energy threshold.

\section{ACKNOWLEDGMENT}

This work was partially funded (Professor O.J. Escalona) by philanthropic funds equally from the Ulster Garden Villages Ltd (UK) and the McGrath Trust (UK).

\section{CONFLict of InTEREst}

The authors declare that they have no conflict of interest.

\section{REFERENCES}

1. Kodoth V, Castro NC, Glover BM, Anderson JMcC, Escalona OJ, Lau E, Manoharan G. Waveform optimization for internal cardioversion of atrial fibrillation. J Electrocardiol, 2011; 44:689-693.

2. Walsh SJ, Manoharan G, Escalona OJ, et al. Novel rectangular biphasic and monophasic waveforms delivered by a radiofrequency powered defibrillator compared with conventional capacitor based waveforms in transvenous cardioversion of atrial fibrillation. Europace, 2006; 8:873880.

3. Eckhard A, Schmitt C, Ammer R, Plewan A, Evans F, Pasquantonio J, Ideker T, Lehmann G, Pütter K, Schömig A. Effect of electrode position on outcome of low-energy intracardiac cardioversion of atrial fibrillation, Am J Cardiol., 1997; 5:621-625.

4. Glover BM, McCann C, Manoharan G,Walsh SJ, Moore MJ, Allen JD, Escalona O, Anderson JMcC, Trouton TG, Stevenson M, Roberts MJ, Adgey JA. A pilot study of a low-tilt biphasic waveform for transvenous cardioversion of atrial fibrillation. PACE - Pacing Clin Electrophysiol, 2008; 31:1020-1024.

5. Walsh PR, Escalona OJ, Kodoth V, McEneaney D, LauE, Manoharan G. Dynamic changes in intracardiac impedance as a predictive marker during internal cardioversion of atrial fibrillation. Computing in Cardiology, 2013; 40:611-614

6. Walsh PR, Rodrigues PA, Velasquez JJ, Waterman N, Escalona OJ. Impedance compensated passive implantable atrial defibrillator. Electronics Letters, 2014; 50 (17): 1192-1193.

7. Escalona $\mathrm{O}$, Kodoth $\mathrm{V}$, Castro $\mathrm{NC}$, et al. Linear variation analysis of intracardiac atrial impedance during internal cardioversion using rectilinear waveforms and energy step up protocol. Computing in Cardiology, 2012; 39:721-724.

8. Goodfellow J, Escalona OJ, Walsh PR, Kodoth V, Manoharan G. Estimation of atrial fibrillatory frequency by spectral subtraction of wavelet denoised ECG in patients with atrial fibrillation. Computing in Cardiology, 2014; 41:329-332.

Corresponding author:

Professor Omar J. Escalona Ulster University, Shore Road, Newtownabbey, United Kingdom. E-mail: oj.escalona@ulster.ac.uk 